Molecular Physics, 1996, Vol. 88, No. 4, 1005-1009

\title{
Coulomb-attenuated exchange energy density functionals
}

\author{
By PETER M. W. GILl†, ROSS D. ADAMSON† and JOHN A. POPLE \\ $\dagger$ Department of Chemistry, Massey University, Palmerston North, New Zealand \\ \$ Department of Chemistry, Northwestern University, Evanston, IL 60208, USA
}

(Received 30 January 1996; accepted 6 February 1996)

\begin{abstract}
Exact and local spin density approximation (LSDA) exchange energy density functionals in the Coulomb-attenuated Schrödinger equation (CASE) approximation are constructed. When expressed as asymptotic series in the attenuation parameter $\omega$, their leading terms are identical. If a Gaussian basis set is used, the terms in the exact series (but not the LSDA series) can be evaluated analytically, i.e. without resorting to quadrature on a grid.
\end{abstract}

\section{Introduction}

In the last few years, interest has been kindled in the existence and implementation of algorithms that can compute the total Coulomb energy of a system of $n$ localized distributions of charge at a computational cost that is only $O(n)$. The availability of such algorithms [1-6] has ramifications for many areas of computational physics but, in particular, it will expand enormously the range of applicability of density functional theory (DFT) [7-9] for, although DFT is recognized as a useful tool for computational chemistry [10], its application to large systems has been hindered by the same Coulom b bottleneck that afflicts Hartree-Fock (HF) theory.

We have recently introduced [6] the Coulomb-attenuated Schrödinger equation (CASE), the approximation that results when each Coulomb operator $1 / r$ in the Schrödinger equation is replaced by the short-range operator erfc $(\omega r) / r$. Erfc is the complementary error function and $\omega$ is a tunable parameter. At first glance, the CASE approximation appears very crude but, for a number of reasons [6], it works surprisingly well in HF and MP2 calculations. It can also be used as a zeroth-order starting point upon which higher-order theories are based. We develop these elsewhere $[11,12]$ and the approach that we take in the present paper is readily extended to these higher approximations.

We must be careful, however, to be consistent. Attenuating the Coulomb interaction will lead to meaningful results only if it is done systematically. Since the Coulomb, exchange and correlation energies all stem from the Coulomb operators in the Schrödinger equation, all three must be attenuated together in any balanced treatment. In methods based on HF theory, such a balance is maintained automatically because Coulomb, exchange and correlation energies are all treated in terms of twoelectron repulsion integrals. This is not true, however, of DFT schemes in which exchange and correlation effects are treated as functionals of the one-electron density. Consequently, in order to perform DFT calculations using the CASE approximation, we need appropriately 'Coulomb-attenuated' density functionals.

Our starting point is the exact expression for the Coulomb-attenuated exchange energy 


$$
K(\omega)=\pi \int_{0}^{\infty} s \operatorname{erfc}(\omega s) \int \rho_{1}^{2}(\mathbf{r}, s) \mathrm{d} \mathbf{r} \mathrm{d} s,
$$

where $\rho_{1}(\mathbf{r}, s)$ is the spherically averaged spinless first-order density matrix. Our derivations follow those in Chapter 6 of the monograph by Parr and Yang [9]. We extend our results to the spin-polarize d electron gas via a standard transformation and use atomic units throughout.

\section{Coulomb-attenuated LSDA exchange functional}

We begin by generalizing the Dirac exchange functional [13] to a form consistent with the CASE approximation. The density matrix for the homogeneous gas is given by

$$
\begin{gathered}
\rho_{1}^{\mathrm{LDA}}(\mathbf{r}, s)=3 \rho(\mathbf{r})\left[\frac{\sin t-t \cos t}{t^{3}}\right], \\
t=k_{F}(\mathbf{r}) s, \\
k_{F}(\mathbf{r})=\left[3 \pi^{2} \rho(\mathbf{r})\right]^{1 / 3} .
\end{gathered}
$$

Substitution of (2)-(4) into (1) yields

$$
K_{\mathrm{LDA}}(\omega)=\frac{3}{4}\left(\frac{3}{\pi}\right)^{1 / 3} \int \rho^{4 / 3}(\mathbf{r}) \int_{0}^{\infty} \operatorname{erfc}\left(\frac{\omega t}{k_{\mathrm{F}}(\mathbf{r})}\right) \frac{4(\sin t-t \cos t)^{2}}{t^{5}} \mathrm{~d} t \mathrm{~d} \mathbf{r},
$$

and integration over $t$ then affords the spin-compensated exchange functional

$$
K_{\mathrm{LDA}}(\omega)=\frac{3}{4}\left(\frac{3}{\pi}\right)^{1 / 3} \int \rho^{4 / 3} F\left(\frac{\omega}{k_{F}}\right) \mathrm{d} \mathbf{r} .
$$

The novel associated function

$$
F(\lambda)=1-\frac{2 \lambda}{3}\left[2 \sqrt{ } \pi \operatorname{erf}\left(\lambda^{-1}\right)-3 \lambda+\lambda^{3}+\left(2 \lambda-\lambda^{3}\right) \exp \left(-\lambda^{-2}\right)\right]
$$

is plotted in figure 1. The corresponding spin-polarize d exchange functional is given by

$$
K_{\mathrm{LSDA}}(\omega)=\frac{3}{4}\left(\frac{6}{\pi}\right)^{1 / 3} \int \rho_{\sigma}^{4 / 3} F\left(\frac{\omega}{2^{1 / 3} k_{F}^{\sigma}}\right) \mathrm{d} \mathbf{r},
$$

where $\sigma$ represents a spin $(\alpha$ or $\beta$ ). One of us (R.D.A.) has implemented (8) within the Q-Chem computer program [14] and the code can perform both self-consistent and non-self-consistent calculations. The small- $\omega$ and large- $\omega$ expansions

$$
\begin{gathered}
K_{\mathrm{LSDA}}(\omega)=\frac{3}{4}\left(\frac{6}{\pi}\right)^{1 / 3} \int \rho_{\sigma}^{4 / 3} \mathrm{~d} \mathbf{r}-\frac{\omega N_{\sigma}}{\sqrt{ } \pi}+\ldots, \\
K_{\mathrm{LSDA}}(\omega) \sim \frac{\pi}{2 \omega^{2}} \int \rho_{\sigma}^{2} \mathrm{~d} \mathbf{r}-\frac{6^{5 / 3} \pi^{7 / 3}}{80 \omega^{4}} \int \rho_{\sigma}^{8 / 3} \mathrm{~d} \mathbf{r}+\ldots,
\end{gathered}
$$

are easily derived and confirm that (8) reduces to the spin-polarized Dirac functional as $\omega \rightarrow 0$. In contrast, note that the leading term for large $\omega$ involes the integral of $\rho^{2}$, rather than $\rho^{4 / 3}$. 


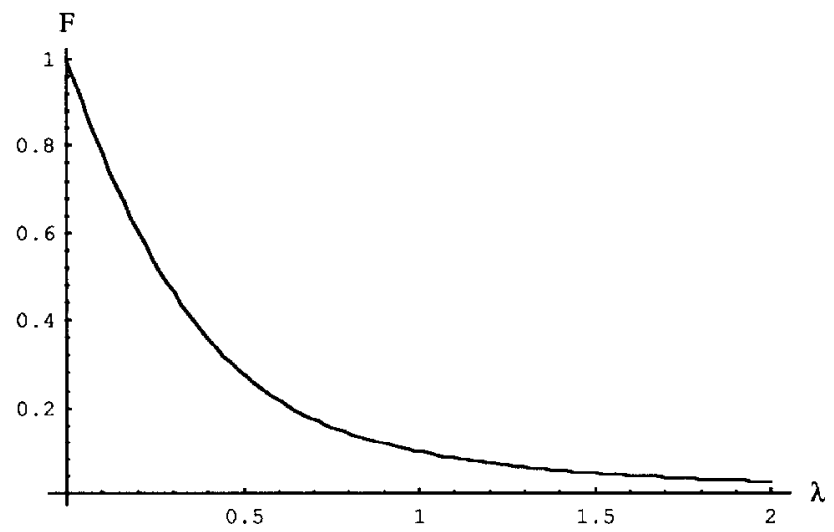

Figure 1. The function $F(\lambda)$ defined by equation (7).

\section{Coulomb-att enuated exact exchange functional}

We next derive the exact exchange functional consistent with the CASE approximation. The density matrix for any inhomogeneous system can be formally expanded [15] as

$$
\begin{gathered}
\rho_{1}^{2}(\mathbf{r}, s)=\rho^{2}(\mathbf{r})-\left[4 \rho(\mathbf{r}) \tau(\mathbf{r})-\rho(\mathbf{r}) \nabla^{2} \rho(\mathbf{r})\right] \frac{s^{2}}{12}+\ldots, \\
\tau(\mathbf{r})=\sum_{i}^{\text {occ }}\left|\nabla \psi_{i}(\mathbf{r})\right|^{2} .
\end{gathered}
$$

Substituting (11) and (12) into (1) easily yields the spin-polarized exact exchange expansion

$$
K_{\sigma}(\omega) \sim \frac{\pi}{2 \omega^{2}} \int \rho_{\sigma}^{2} \mathrm{~d} \mathbf{r}-\frac{\pi}{32 \omega^{4}} \int\left[4 \rho_{\sigma} \tau_{\sigma}+\left|\nabla \rho_{\sigma}\right|^{2}\right] \mathrm{d} \mathbf{r}+\ldots
$$

Because the spin orbitals $\psi_{i}(\mathbf{r})$ appear in (13), it is not a density functional in the purest sense. Nonetheless, practical density functionals containing $\tau(\mathbf{r})$ are becoming increasingly common and, in particular, have recently been cleverly exploited by Becke [16].

Intriguingly, the leading terms in (10) and (13) are identical, from which we deduce that the LSD exchange approximation is exact when the Coulomb operator is sufficiently attenuated. This is a rather surprising conclusion for it is true even of highly inhomogene ous systems.

Before leaving the exact exchange expansion (13), we should make one further remark. The leading term in the asymptotic series (10) and (13) involves the integral of $\rho^{2}$. This is very significant computationally because, if a Gaussian orbital basis set is used, this integral (unlike that of $\rho^{4 / 3}$ ) can be evaluated analytically and efficiently without resort to numerical quadrature. Furthermore, although they become progressively more complicated, all of the terms in (13) can be computed in this way. Our exact exchange expansion is a gridless density functional. Curiously, this is not true of the LSDA exchange expansion (8), (9) or (10).

\section{The hydrogen atom}

To illustrate the behaviour of the two new functionals, we consider the ground state of the $\mathrm{H}$ atom, the simplicity of which permits its exchange energy to be written 


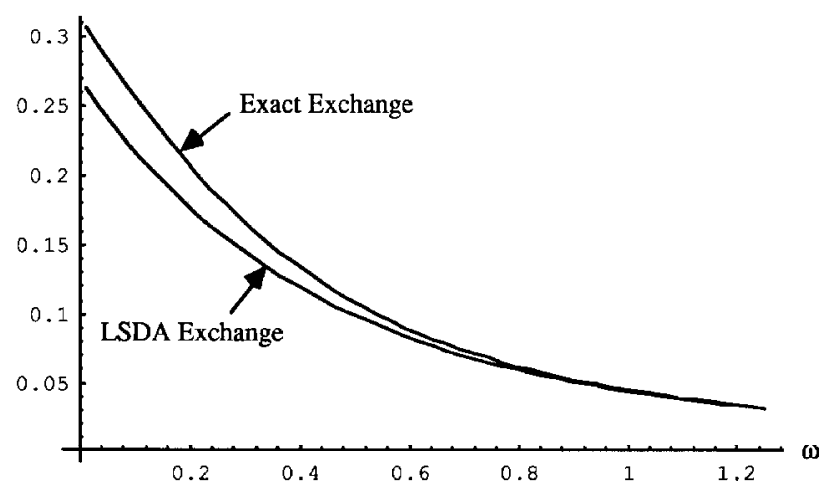

Figure 2. The exact and LSDA exchange energies of the Coulomb-attenuated H atom.

in closed form. The atom's exact density is $\rho(r)=\exp (-2 r) / \pi$ and, in a Coulombattenuated framework, its exact exchange energy is therefore given by

$$
\begin{aligned}
K(\omega) & =\frac{1}{2} \iint \rho\left(r_{1}\right) \frac{\operatorname{erfc}\left(\omega r_{12}\right)}{r_{12}} \rho\left(r_{2}\right) \mathrm{d} \mathbf{r}_{1} \mathrm{~d} \mathbf{r}_{2} \\
& =\frac{5}{16}-\left(\frac{5}{16}-\frac{3}{8 \omega^{2}}+\frac{1}{4 \omega^{4}}-\frac{1}{6 \omega^{6}}\right) \exp \left(1 / \omega^{2}\right) \operatorname{erfc}(1 / \omega)-\left(\frac{5}{8 \omega}-\frac{1}{3 \omega^{3}}+\frac{1}{6 \omega^{5}}\right) \frac{1}{\sqrt{ } \pi} .
\end{aligned}
$$

This is the upper curve in figure 2. As would be expected on physical grounds, the energy decays smoothly from its unattenuated value of $5 / 16$ as the parameter $\omega$ is increased. We have also found the Coulomb-attenuated LSDA exchange energy by substituting the $\mathrm{H}$ atom density into (8) and evaluating the $\mathbf{r}$ integral numerically. This produces the lower curve in figure 2 .

At $\omega=0$ (the unattenuated limit), the exact and LSDA energies are 0.3125 and $0 \cdot 2680$, respectively, illustrating the rule of thumb that the LSDA underestimates exchange energies by roughly $10 \%$. It is clear from figure 2 , however, that these energies approach one another quite rapidly as $\omega$ increases and are almost identical beyond $\omega=1$, suggesting that (10) and (13) may be more similar than they appear superficially. Indeed, the asymptotic series

$$
\begin{gathered}
K_{\mathrm{LSDA}}^{\mathrm{H}}(\omega) \sim \frac{1}{16 \omega^{2}}-\frac{1}{35 \cdot 698 \omega^{4}}+\ldots, \\
K^{\mathrm{H}}(\omega) \sim \frac{1}{16 \omega^{2}}-\frac{1}{32 \omega^{4}}+\ldots,
\end{gathered}
$$

obtained from (10) and (13) using the $\mathrm{H}$ atom density, confirm that the LSDA is a surprisingly accurate exchange approximation in this (highly inhomogeneous) atom, even for rather modest values of $\omega$.

We thank Dr Benny Johnson for stimulating discussions during his visit to Massey University. This research was supported in part by Q-Chem Inc., the US National Science Foundation (Grant DMI-9460396) and the New Zealand Lottery Science Board (Grant 45088). One of us (R. D. A.) gratefully acknowledges a Ph.D. scholarship from Massey University. 


\section{References}

[1] Greengard, L., and Rokhlin, V., 1987, J. comput. Phys., 73, 325.

[2] White, C. A., Johnson, B. G., Gill, P. M. W., and Head-Gordon, M., 1994, Chem. phys. Lett., $230,8$.

[3] Kutteh, R., Aprà, E., and Nichols, J., 1995, Chem. phys. Lett., 238, 173.

[4] Strain, M. C., Scuseria, G. E., and Frisch, M. J., 1996, Science, 271, 51.

[5] Dombroski, J. P., Taylor, S. W., and Gill, P. M. W., 1996, J. phys. Chem., in press.

[6] Adamson, R. D., Dombroski, J. P., and Gill, P. M. W., 1996, Chem. phys. Lett., in press.

[7] Hohenberg, P., and Kohn, W., 1964, Phys. Rev. B, 136, 864.

[8] Kohn, W., and Sham, L. J., 1965, Phys. Rev. A, 140, 1133.

[9] Parr, R. G., and YANG, W., 1989, Density-Functional Theory of Atoms and Molecules (Oxford: Clarendon).

[10] Johnson, B. G., Gill, P. M. W., and Pople, J. A., 1993, J. chem. Phys., 98, 5612.

[11] Adamson, R. D., Dombroski, J. P., and Gill, P. M. W., 1996, Chem. phys. Lett., in preparation.

[12] Dombroski, J. P., Taylor, S. W., and Gill, P. M. W., 1996, J. chem. Phys., in preparation.

[13] Dirac, P. A. M., 1930, Proc. Camb. Phil. Soc., 26, 376.

[14] Johnson, B. G., Gill, P. M. W., Head-Gordon, M., White, C. A., Maurice, D. R., Adamson, R. D., Adams, T. R., and Oumi, M., 1996, Q-Chem (Pgh, USA: Q-Chem Inc.).

[15] Berkowitz, M., 1986, Chem. phys. Lett., 129, 486.

[16] Becke, A. D., 1996, J. chem. Phys., 104, 1040. 
